\title{
Impact de la maréchalerie sur le pied du cheval (Cas du Cercle Hippique de Lubumbashi)
}

\author{
Binemo K.Jean Claude, Lenge M.N.Moïs, Binemo M.Clement. E Lumbu B. Christelle \\ Haut-Katanga, Université de Lubumbashi 1825 Lubumbashi Kinshasa, RDC \\ Auteur Correspondant drbinemofils@gmail.com
}

Original submitted in on 31st May 2018. Published online at www.m.elewa.org on $30^{\text {th }}$ September 2018 http://dx.doi.org/10.4314/jab.v129i1.9

\section{RÉSUMÉ}

La valeur du cheval dépend de l'état de ses membres et surtout de ses pieds. La maréchalerie représentée par le ferrage et le parage, constitue le moyen le plus usuel pour entretenir ses pieds. Cette étude décrit les symptômes, les lésions et les anomalies dus à la maréchalerie observés chez les chevaux du cercle hippique de Lubumbashi $(\mathrm{CHL})$ avant de proposer certains détails techniques et scientifique.

L'observation rigoureuse de ces derniers pourrait faire de la maréchalerie un travail d'orfèvre au cercle hippique de Lubumbashi.

Mots clés : Maréchalerie, cheval, pied, anomalies, cercle hippique, Lubumbashi

\section{ABSTRACT}

The value of the horse depends on the state of its limbs and especially on its feet. The blacksmithing, represented by the shoeing and trimming, is the most common way to maintain its feet. This study describes the symptoms, lesions and anomalies due to the blacksmithing observed in the horses of the Lubumbashi Equestrian Circle $(\mathrm{CHL})$ before proposing certain technical and scientific details.

Keywords : blacksmithing, horse, foot, anomalies, equestrian circle,

\section{INTRODUCTION}

Le cheval est un animal de travail dont la valeur dépend de l'état de ses membres et surtout de ses pieds (Collin, 2005). C'est ainsi qu'un adage dit que: ( pas de pieds, pas de cheval ) (Castelijns, 2003 ; Hennig, 2003) Pour tous les animaux, la vie à l'état sauvage maintient les pieds dans leur conformation normale ce qui n'est pas le cas avec la domestication, car le service impose aux animaux et leur hygiène de vie sont rarement en concordance avec les impératifs physiologiques, d'où l'apparition d'une pathologie de l'ongle qui entraine par ricochet une science de l'entretien de ce dernier. Pour le cheval, cette science c'est la maréchalerie avec sa technique la plus usuelle : la ferrure (Collin, 2003). En outre, le pied constitue la principale zone des pathologies du cheval de sport dont $80 \%$ des lésions qui se recrutent au niveau des antérieurs (Amelineau, 2004). Le présent travail vise à relever l'impact de la maréchalerie au Cercle Hippique de Lubumbashi (CHL), sur les problèmes survenant aux pieds des chevaux de cette entité sportive. 

Lubumbashi)

\section{MATERIELS ET METHODE}

Milieu : Le Cercle Hippique de Lubumbashi (CHL) est une association sans but lucratif (asbl), créée en 1967. Le $\mathrm{CHL}$ regroupe les cavaliers et les propriétaires des chevaux qui ont manifesté le souci de maintenir les habitudes et l'épanouissement de l'équitation. Le cercle hippique de Lubumbashi (CHL en sigle) est une association sans but lucratif créée en 1967 qui regroupe les cavaliers et les propriétaires des chevaux. II vise essentiellement la pratique, l'amélioration et le développement de l'équitation sportive ainsi que le maintien de la tradition de cavalerie chez les cavaliers. II est situé à l'Ouest de la ville de Lubumbashi. Celle-ci appartient à la zone climatique tropicale humide de type CW6 selon la classification de KÖPPEN. Le cercle hippique de Lubumbashi est limité au Nord-ouest par la cité Karavia ; au Sud par la cité Gécamines, le quartier Penga-penga et le quartier Gbadolite, à l'Est par le quartier Kabulameshi et au Nord par le nouveau lotissement (quartier Kabulameshi). Cette ASBL vise essentiellement : la pratique de l'équitation sportive dans notre pays, l'amélioration et le développement de l'équitation, le maintien chez le cavalier des traditions de la cavalerie ainsi que l'honneur, la loyauté et l'esprit sportif. Pour une meilleure exploitation des chevaux, le cercle hippique de Lubumbashi comprend les infrastructures ci-après :

Des prairies dont certaines sont clôturées avec des fils de fer et d'autres clôturées avec des planches de bois. Ces prairies où l'on enferme les chevaux à certaines heures de la journée, permettent à ces dernières de brouter de l'herbe en dehors de la ration distribuée dans les boxes;

- $\quad$ Des carrières dont l'une contient une pelouse sur laquelle sont organisés les concours hippiques, les autres contenant le sable ou les scories qui servent aux entraînements ;

- Une infirmerie, un dépôt d'aliment, une sellerie, des boxes des chevaux un bar et quelques maisons d'habitations pour les travailleurs.

Animaux : Notre étude a porté sur 43 chevaux dont 11 poneys et 32 chevaux de race pur-sang anglais et demisang. Selon le sexe, ces animaux sont repartis comme suit : 2 étalons, 10 juments et 31 hongres ; tous adultes et un seul présentant un mauvais état général.

Ces chevaux ont été suivis de novembre 2009 à Mai 2010.

Matériel : Le matériel ci-dessous a été utilisé pour réaliser le ferrage, le déferrage, le parage et / ou le traitement médical chez les animaux à savoir :

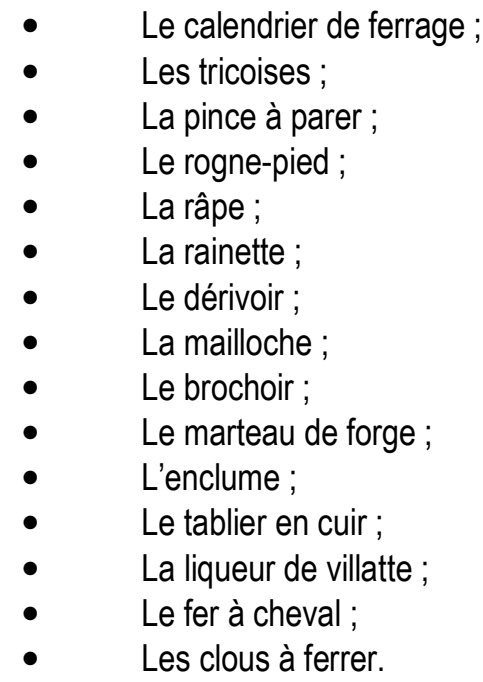

\section{Méthodes}

Consultation du calendrier : Avec le maréchal-ferrant, on consultait le calendrier de ferrage / parage du mois en cours pour répertorier les chevaux prévus.

Examen des aplombs : Les chevaux sélectionnés sont amenés tour à tour dans le box de ferrage pour l'examen des aplombs afin de se faire une idée exacte sur leur stature pour un meilleur parage associé ou non au ferrage.

Déferrage: Pour retirer le fer le maréchal-ferrant commence par révéler et couper les rivets des clous à l'aide du dérivoir et de la mailloche. Ensuite, le maréchalferrant introduit les mors des tricoises sous l'extrémité d'une des branches du fer et appuie vers le bas pour soulever la branche du fer pour dégager le fer du pied. Après, il agit de même pour tous les pieds portant des fers. Si des morceaux de clous restent dans la corne, ils sont chassés à l'aide du dérivoir.

Les fers sont déclassés après trois ferrages suite à leur usure.

Parage : Les chevaux non ferrés sont parés toutes les cinq à six semaines, mais il arrive souvent que des chevaux aient besoin d'être parés avant que ce délai se soit écoulées lorsque la corne a trop poussé ; ce qui est le cas pendant la saison des plaies. Le parage commence par un nettoyage du pied notamment la muraille, la sole, la fourchette en enlevant les parties mortifiées soit avec la rainette soit avec le rogne-pied et la mailloche. L'excès de corne est coupé à l'aide de la pince à parer ou le rogne-pied et la mailloche, en commençant par la pince pour remontrer vers les talons. Ensuite, la sole est nettoyée en enlevant les parties mortifiées toujours à l'aide du rogne-pied et de la 
mailloche. Enfin, le maréchal-ferrant redonne au pied sa forme à la râpe. A l'occasion du parage, le maréchalferrant repère certaines pathologies comme les seimes, les bleimes, les pourritures de la fourchette,

Ferrage ou reformage : Au Cercle Hippique, on travail selon la méthode anglaise et pour cela, le maréchalferrant pose le fer sur le pied et examine si les contreperces en pince sont au niveau de la ligne blanche. II place d'abord les clous en pince en les chassant à coup

\section{RÉSULTATS}

Sur un total de 43 chevaux suivis, seuls 11 ont présenté des problèmes au parage et au ferrage. La situation générale de ferrage chez les chevaux suivis est présentée dans le tableau 1 les anomalies particulières de brochoir, puis les clous en talons enfin les autres clous. Après avoir placé les clous, le maréchal-ferrant pratique une encoche en dessous de la lame de chaque clou à l'aide de la râpe ; il coupe les bouts de la lame des clous avec les tricoises et rabat les rivets dans les encoches à l'aide de la pince à river. Pour terminer, il redonne un dernier coup de râpe et examine si les quatre pieds sont bien d'aplombs.

observées sur certains d'entre eux sont reprise dans le tableau 2; tandis que les symptômes ou lésions observées chez les sujets ferrés ou non ferrés dans le tableau 3.

Tableau 1 : État de la maréchalerie chez les chevaux du CHL

\begin{tabular}{c|c|c|c|c}
\hline \multirow{1}{*}{ Sexe } & \multicolumn{2}{|c|}{ Chevaux ferrés } & Chevaux non ferrés & Total \\
\cline { 2 - 3 } & Antérieurs seulement & Antérieures et postérieurs & & \\
\hline Étalons & 0 & 2 & 0 & 2 \\
Juments & 1 & 1 & 8 & 10 \\
Hongres & 5 & 15 & 11 & 31 \\
$\quad$ Total & $\mathbf{6}$ & 18 & 19 & $\mathbf{4 3}$ \\
\hline
\end{tabular}

A l'observation de ce tableau, il ressort que $55,8 \%$ des chevaux étaient ferrés dont $41,9 \%$ aux quatre membres et 13,9 $\%$ aux antérieurs seulement.

Tableau 2 : Anomalies observées sur les chevaux étudiés

\begin{tabular}{l|l|c|c}
\hline $\mathbf{N}^{\circ}$ & Sexe & Anomalies & Autres observations \\
\hline & & & \\
\hline 1. & Jument & - & Non ferré \\
\hline 2. & Jument & - & Non ferré \\
\hline 3. & Hongre & - & Ferré \\
\hline 4. & Hongre & - & Non ferré \\
\hline 5. & Hongre & - & Fon ferré \\
\hline 6. & Hongre & - & Ferré \\
\hline 7. & Hongre & - & Ferré \\
\hline 8. & Hongre & - & Ferré \\
\hline 9. & Hongre & - & Ferré \\
\hline 10. & Hongre & Membres Cagneux & Ferré \\
\hline 11. & Hongre &
\end{tabular}

II ressort de ce tableau que la majorité $(81,8 \%)$ de chevaux suivis et qui ont présenté des problèmes sont des hongres et le sabot non pigmenté constitue la majeur anomalie observée tant chez les sujets ferrés que non ferrés comparativement aux membres panards $(0,9 \%)$ et cagneux $(0,9 \%)$. 
Binemo et al., J. Appl. Biosci. 2018 Impact de la maréchalerie sur le pied du cheval (Cas du Cercle Hippique de Lubumbashi)

Tableau 3 : Symptômes ou lésions observés chez les chevaux ferrés et non ferrés

\begin{tabular}{c|l|l|l|l}
\hline $\mathbf{N}^{\circ}$ & Symptômes & Causes & Traitement & observation \\
\hline 1. & Légère boiterie & $\begin{array}{l}\text { Parage } \\
\text { excessif }\end{array}$ & - & Disparition spontanée des symptômes \\
\hline 2. & Rougeur de la ligne blanche & $\begin{array}{l}\text { Parage } \\
\text { excessif }\end{array}$ & - & Disparition spontanée de la lésion \\
\hline 3. & $\begin{array}{l}\text { Légère boiterie, rougeur de } \\
\text { La ligne blanche }\end{array}$ & $\begin{array}{l}\text { Parage } \\
\text { excessif }\end{array}$ & - & Disparition spontanée des symptômes \\
\hline 4. & $\begin{array}{l}\text { Légère boiterie, rougeur de la } \\
\text { ligne blanche }\end{array}$ & $\begin{array}{l}\text { Parage } \\
\text { excessif }\end{array}$ & - & Disparition spontanée des symptômes \\
\hline 5. & $\begin{array}{l}\text { Légère boiterie, rougeur de la } \\
\text { ligne blanche }\end{array}$ & $\begin{array}{l}\text { Parage } \\
\text { excessif }\end{array}$ & - & Disparition spontanée des symptômes \\
\hline 6. & Boiterie légère & Clou serrant & Retrait du clou & $\begin{array}{l}\text { Disparition spontanée des symptômes } \\
\text { après retrait du clou }\end{array}$ \\
\hline 7. & Boiterie légère & Clou serrant & Retrait du clou & $\begin{array}{l}\text { Disparition spontanée des symptômes } \\
\text { après retrait du clou }\end{array}$ \\
\hline 8. & Boiterie légère & Clou serrant & Retrait du clou & $\begin{array}{l}\text { Disparition spontanée des symptômes } \\
\text { après retrait du clou }\end{array}$ \\
\hline 9. & Boiterie légère & Clou serrant & Retrait du clou & $\begin{array}{l}\text { Disparition spontanée des symptômes } \\
\text { après retrait du clou }\end{array}$ \\
\hline 10. & Forte boiterie & $\begin{array}{l}\text { Piqûre par } \\
\text { un clou }\end{array}$ & $\begin{array}{l}\text { Retrait du clou, } \\
\text { traitement local à } \\
\text { la liqueur de } \\
\text { villatte }\end{array}$ & $\begin{array}{l}\text { Disparition des symptômes après trois } \\
\text { jours }\end{array}$ \\
\hline 11. & Forte boiterie & $\begin{array}{l}\text { Piqûre par } \\
\text { un clou }\end{array}$ & $\begin{array}{l}\text { Retrait du clou, } \\
\text { traitement local à } \\
\text { la liqueur de } \\
\text { villatte }\end{array}$ & $\begin{array}{l}\text { Disparition des symptômes après un } \\
\text { jour }\end{array}$ \\
\hline
\end{tabular}

A l'observation de ce tableau, il ressort que le parage excessif et le mauvais emplacement des clous ont été à l'origine des boiteries observées après parage et ferrage.

\section{DISCUSSION}

La présente discussion portera sur l'état de la maréchalerie, les anomalies observées sur les chevaux, la technique de ferrage / parage appliquée ainsi que sur les résultats obtenus au $\mathrm{CHL}$.

État de la Maréchalerie: Au cercle hippique de Lubumbashi, plus de la moitié des chevaux $(55,8 \%)$ sont ferrés (tableau 1). En effet, le fer étant une sorte de semelle de protection pour le sabot, il prévient l'usure excessive de la corne et son éclatement. II s'agit donc d'une prévention contre une usure trop rapide ou irrégulière de la corne chez les chevaux suite au travail auquel ils sont soumis. Sans cette protection, les pieds s'exposeraient aux dommages suite aux efforts qui leur sont demandés et la paroi s'userait de telle façon que le pied reposerait sur la sole et dans ce cas les irrégularités du sol provoqueraient de graves problèmes, notamment des contusions (Ndour, 2010 ; Rose et Hodgson, 2004). Toutefois, selon certains observateurs, les chevaux libres de leurs mouvements et se nourrissant à volonté développent une sorte d'autocorrection de leur aplomb. Par ailleurs, en le gardant dans un box 20 heures sur 24 et en les forçant à travailler sur divers terrains, on dérègle ce système d'autocorrection. II s'avère alors nécessaire de parer voire l'obligation de ferrer le cheval en vue de soulager les parties latérales ou médiales selon le défaut de conformation et de l'utilisation qu'on en fait et surtout le préserver dans le temps (Demoix, 2000). Dans cette optique, le ferrage devient non plus un " mal nécessaire » comme on le dit trop souvent mais plutôt " un fait utile » s'il est exécuté selon les besoins biomécaniques du pied. Parmi les chevaux ferrés, la majorité $(41,9 \%)$ étaient ferrés aux quatre membres et le reste $(13,9 \%)$ ne l'étaient 

Lubumbashi)

qu'aux antérieurs. En effet, les antérieurs supportant plus de poids que les postérieurs et de ce fait, leurs sabots s'usent plus vite. Lorsque le cheval ne travaille que sur du sable ou quand il est au repos ou en pâture, il n'est forcément pas utile de ferrer les postérieurs mais il vaut mieux continuer à protéger les antérieurs (Demoix et Crevier-Demoix, 2003). II est ressort de cette étude que parmi les chevaux ferrés, la majorité était des hongres $(46,5 \%)$, les juments et les étalons ne représentant qu'équitablement $4,6 \%$. Cette répartition ne se justifie que par la supériorité numérique des hongres dans l'exploitation et leur appartenance soit au propriétaire soit au cercle. Par ailleurs, 44,\% des chevaux élevés au cercle hippique de Lubumbashi, ne sont pas ferrés. Ils comprennent 8 juments et 11 hongres. En effet, d'après Webber (2002), le fer ne s'impose pas si les pieds sont de bonne conformation (durs, corne solide, fourchette bien développée). De même si un cheval est amené à ne travailler que sur des surfaces douces, ses pieds n'auront pas besoin d'être protégés. Bien que ce manque de ferrage ait pour avantage la réduction du coût d'entretien du cheval, les pieds non ferrés doivent cependant être parés toutes les six semaines au plus. En plus, si les chevaux mis au pré ensemble se donnent des coups de pieds, les pieds non ferrés, en particuliers les postérieurs feront moins de dégât que les ferrés.

Anomalies : Les membres panard $(0,9 \%)$ et cagneux $(0,9 \%)$ n'ont été révélés que sur deux hongres (Tableau 2). D'après Vanschepdael (2003), le cheval cagneux contrairement au cheval panard a les pinces des deux pieds convergents vers l'avant. Pendant la phase de soutien, le pied dévie sa trajectoire vers l'extérieur. Cette complication de la conformation peut provoquer des interférences notamment au niveau du boulet, de l'os sésamoïde. Par ailleurs, il renchérit en stipulant que les défauts d'aplomb sont courants et souvent négligés. Ils ont pourtant des conséquences sur les articulations des jambes et également dans la partie supérieur du corps (épaule, encolure, épine dorsale). Quant aux chevaux panards et serrés du devant, ils peuvent rarement supporter un travail intensif. Cette anomalie constitue une des pires défectuosités d'aplomb des membres antérieurs (Vanschepdael, 2003). Les défectuosités d'aplomb interviennent aussi dans la production des boiteries du bas et notamment, en entraînant l'apparition de production ostéophytes au niveau de l'insertion des ligaments (Hodson et al., 2000).

Ferrage et Parage : D'après Spriet (2002), tout ferrage doit commencer par un bon parage qui est la taille de la corne avant la pose du fer. Cette opération est nécessaire afin de retire l'excès de corne qui pousse régulièrement de l'ordre de $1 \mathrm{~cm}$ tous les mois. Le ferrage redonne ainsi au pied son aplomb normal. On reconnaît un cheval correctement paré si la pince est dans le prolongement du paturon, de face et de profil. En outre, un bon parage est essentiel pour éviter l'apparition d'arthrose ou de tendinites diverses mais un bon fer ne pourra jamais compenser un mauvais parage. Au cercle hippique de Lubumbashi, le ferrage se déroule selon la méthode anglaise c'est-à-dire que le maréchal-ferrant le réalise seul et à froid. Cette méthode a l'avantage d'être économique et de permettre au maréchal-ferrant d'avoir une bonne vision du sabot. Contrairement à la méthode française dans laquelle, le maréchal-ferrant se fait seconder par un aide et à la ferrure au travail considéré comme coercitive et réservé aux cas difficiles (Vanschepdael, 2003 et Castelijns, 2003). Le ferrage peut se réaliser à chaud ou à froid, la corne n'étant ni innervée ni vascularisée; ce qui permet une application indolore de la ferrure même à chaud, sans oublier que le pododerme peut souffrir de la rétraction du fait de l'irradiation de la chaleur. Cependant, il est préférable d'opter pour un ferrage à froid tout aussi solide. Au cercle hippique de Lubumbashi, le renouvellement de ferrure intervient après 5 à 6 semaines; ceci corrobore Ndour (2010) et Spriet (2002) qui fixent le rythme habituel de renouvellement de fer entre 4 et 6 semaines, tandis que pour les chevaux de course, il est fixé a 2 à 3 semaines, pas toujours en fonction de leur usure mais en fonction de la pousse de la corne.

Symptômes et/ou lésions observées: Une légère boiterie consécutive au parage excessif a été observée chez 3,63\% de chevaux suivis. En effet, d'après Hennig (2003) ; Amelineau (2004) ; Binemo (2007), le parage consiste à rendre aussi normal que possible la forme, l'inclinaison et l'aplomb du pied. C'est pour cette raison que le maréchal-ferrant doit aussi bien éviter un parage insuffisant qu'un parage excessif qui sera à l'origine d'une hypersensibilité du pied et d'éventuelles blessures profondes (Castelijns, 2003). En outre, un parage insuffisant est souvent à l'origine de la déformation du sabot, laquelle peut entraîner un mauvais appui sur le sol et amener à moyen terme des défauts de locomotions (Hodson, 2000). D'après Guillaume (2001), le brochage des clous doit être réalisé de sorte que les lames sensibles du pied ne soient pas touchées et que les clous doivent être plantés à l'extérieur de la ligne blanche pour ressortir sur la paroi sinon, on parlerait de clous serrant lesquels peuvent être à l'origine des boiteries pourquoi pas d'infection lorsque non décelées le plus tôt possible. Par ailleurs, cette légère boiterie observée a été en général accompagnée de la rougeur de la ligne blanche 
$(2,72 \%)$, ceci serait dû non seulement au mauvais brochage du fer tel qu'élucidé ci haut mais aussi à des éventuelles plaies ou infections légères de cette structure qui anatomiquement sépare les tissus durs des tissus mous du pied (Denoix, 2000 ; Amelineau, 2004). II a été également observé une forte boiterie due à la piqûre par un clou. D'après Hennig (2003), le clou ou tout autre objet

\section{CONCLUSION}

II ressort de notre étude que la maréchalerie telle que pratiquée au Cercle Hippique de Lubumbashi, correspond du point de vue technique aux normes scientifiquement admises et recommandées par plusieurs auteurs, notamment ceux qui considèrent le ferrage non pas comme un "mal nécessaire " mais un "fait utile " (Guillaume, 2001). Au cercle hippique de Lubumbashi, le cheval cagneux et le cheval panard constituent les seuls anomalies observées sur quelques chevaux. Le ferrage

\section{BIBLIOGRAPHIE}

Amelineau L. Le pied du cheval : anatomie, aplombs, maréchalerie, pathologie, Thèse: Méd Vét: Lyon (Université Claude Bernard), 2004.

Binemo M.C. : Cours de Chirurgie spéciale des Grands et Petits Animaux, 2ieme doctorat, Médecine Vétérinaire, UNILU, 2007 ;

Castelijns H. Soins du pied adaptés à la fourbure. Infor Maréchalerie $\mathrm{N}^{\circ} 100,2003$.

Collin B. Anatomie du cheval, Liège : Éditions Derouaux Ordina, 690p, 2005.

Denoix J.M. The equine distal limb, An atlas of clinical anatomy and comparative imaging, Londres Manson Publishing, 390p, 2000.

Denoix JM., Crevier-Denoix N. Les lésions tendineuses, infor Maréchalerie, Vol 103, 8-18, 2003.

Guillaume O. Le pied du cheval : cd-rom présentant son anatomie et les bases de maréchalerie, Thèse : Méd Vét Université Claude Bernard Lyon, 70p, 2001.

Hennig F. L'exemple NBS. L'utilisation des nouvelles théories, techniques et matériaux. Infor Maréchalerie, Vol 102, 56-62, 2003.

Hodson E., Clayton H.M. et Lanovaz J.L. The forelimb in walking horses: 1. Kinemetics and ground reaction forces. Equine Veterinary Journal, Vol 32(4) 287-294, 2000.

Ndour M. Caractérisation Biomédicale et les principales pathologies du pied du cheval de trait dans la région de Dakar, Thèse : Méd Vét, Dakar ; 29 , 126p, 2010. pointu, qui traverse la sole, si elle est mince et desséchée, est susceptible d'entraîner les plaies profondes. Toutefois, il est toujours conseillé de s'assurer que la boiterie n'est pas causée par un clou de ferrure ou une infection de la ligne blanche parce que ces affections ont les mêmes symptômes et les mêmes altérations pathologiques que les plaies profondes (Collin, 2005).

ou le déferrage intervenant après parage à des intervalles réguliers font de la maréchalerie une véritable préoccupation tant de la part du président du cercle, du vétérinaire, des moniteurs d'équitation et des propriétaires des chevaux que du maréchal-ferrant. En dépit des faibles pourcentages de boiteries observés dans le résultat, le ferrage à l'anglaise devrait être correctement réalisé, faisant ainsi de la maréchalerie un travail d'orfèvre au $\mathrm{CHL}$.

Rose R.J. et Hodgson D.R. Manuel de la pratique équine 2ème éd: Oxford, 768p, 2004.

Spriet M. Diagnostic différentiel des boiteries chroniques de la région palmaire du pied chez le cheval, Thèse : Méd Vét : Université Claude Bernard, Lyon 102p, 2002.

Vanschepdael P. Effets biomécaniques d'une augmentation de la base d'appui postérieure $\mathrm{du}$ fer. Infor Maréchalerie, Vol 102, 48-54, 2003.

Webber Tony, Pied et Bouche, éd. Proxima, Chamalières, 2002. 